\title{
e-Learning Technology: Convergence with the Mainstream
}

\author{
Colin Harrison \\ IBM Institute for Advanced Learning, IBM Zürich Research Laboratory, 8803, Rüschlikon, \\ Switzerland
}

ch@zurich.ibm.com

Key words: Learning Objects, Sequencing, Interaction Modelling, Web Services

\begin{abstract}
The evolution of technology-based learning systems over the last thirty years has been driven by the desire to implement increasingly rich pedagogical models. This has led to remarkable innovations in interaction, collaboration, and the use of rich-media in learning systems. However, these technology innovations have rarely been absorbed into the mainstream of information technology and consequently have failed to evolve into broadly implemented standards. Abstractly we can view learning as a sequence of encounters among instructors, learners, and information. These encounters may be synchronous or asynchronous, local or distant, formal or informal, solitary or en masse. Computer Science is seeking to develop technologies that will support a new set of Web experiences. These technologies will provide a generalised model for describing encounters among people and information and are based on open industry standards. The application space for these experiential technologies will eventually include e-commerce, professional communications, games and entertainment, but learning appears likely to be the first practical application.
\end{abstract}

\section{INTRODUCTION}

For over 20 years we have been installing personal computers in classrooms for primary, secondary, and tertiary education in the deeply

The original version of this chapter was revised: The copyright line was incorrect. This has been corrected. The Erratum to this chapter is available at DOI: 10.1007/978-0-387-35663-1_34 
rooted belief that this will improve education. While the computer is clearly an important tool for 21C knowledge workers, this has been true of many $20 \mathrm{C}$ inventions that have not been so strongly embraced. Moreover, today at least, many learners have better access to computers at home than at school or university. There is something intensely compelling about the personal computer that convinces many people that it must a priori be a valuable tool for learning.

Some speak of technology-based learning - hereafter e-Learning - as the manifest destiny for the transformation of our educational systems and then seek to address "obstacles" to this transformation. This perspective ignores the distress in educational systems and prompts the question: If e-Learning is such a powerful technique, what critical problems in training and education can it solve? Practitioners lack professional development opportunities to get beyond the practicalities of how to drive a Web browser and into a deeper understanding of how this technology will inevitably transform educational methods. These two communities need much deeper engagement to take our instinctive belief in the educational value of personal computers to a level where we can talk factually about the relative merits of investment in e-Learning versus investment in other education facilities.

In the work of IBM's Institute for Advanced Learning, we view the computer as an active partner in a dialogue that includes instructor, students, mentors, and other parties. We view the learning process as a collection of encounters between the individual student and the instructor, the body of knowledge, other students, and a variety of learning methods including discovery, inquiry and apprenticeship. The instructor orchestrates these encounters to enable each student to reach the desired learning outcome. We view the computer as a facilitator for many of these encounters, adding to the traditional classroom experience the benefits of Information and Communication Technologies (ICT). The instructor should orchestrate this facilitation as part of the overall learning process.

Our key goals are:

- to provide leadership to educators and industry in developing a set of technologies and content based on open industry standards to fully develop the capabilities of e-Learning;

- to work towards a factual basis for the application of these capabilities to produce specific learning outcomes.

The result should be that educational practitioners extend their learning methods in pedagogically sound ways supported by tools and content that are meaningful in the learning context.

These are extremely large and complex problems so we seek key leverage points where IBM is well placed to produce movement towards these goals. 


\section{HOW CAN COMPUTERS HELP LEARNING?}

Let's start with a simple question. In what ways do we think computers can be helpful and add value in learning? This has been widely debated and the answers suggest it has something to do with:

- access to information, both local and also beyond the boundaries of the learning institution;

- structuring frameworks for interactions among instructors, learners, and tutors;

- enabling communication among a group of people who cannot be at the same place at the same time;

- interaction with simulations and games, enabling new forms of learning experiences;

- individualised learning;

- new methods of learning \& assessment;

- providing communities of interest for instructors, parents, and learners. Note that we are not looking at the computer as a vehicle for pedagogy. This role falls to the instructor, who may employ the computer to support various activities within a learning method.

How does the computer contribute to these activities - what high-level capabilities of the computer support these? Here is a partial list:

- Web browsing and browsing other documents, whether local or remote, the ability to access and experience digital information.

- Multimedia, the ability to deliver digital information in the form of moving pictures and sounds.

- Interaction, the ability of the computer to modify its actions in response to input from the user. This capability is basic to all forms of learning experiences. A specific form of interaction is the ability of the computer to modify its actions based on a simulated interaction with a virtual environment, such as a flight simulator, or with a human partner, such as an injured patient. Closely related to these interactions are games, where players work (competitively or collaboratively) towards a goal.

- Adaptation, the ability of the computer to adapt its interaction with the user to optimise the experience it provides - in terms of learning or completing a transaction - to the user's needs.

- Collaboration, the ability of a group of people to have common views of documents, multimedia content, and chat. Examples include one-on-one dialogues for learning and coaching, collaborative problem solving, and guided use of a computer application. 
- Authoring, the ability of the computer to manipulate multimedia and other content at the direction of a user, typically an instructor, to produce a meaningful learning object. At its most basic this is the capture of primitive content - text, image, objectives or state. At its most advanced it may begin to provide pedagogical support for specific learning goals.

- Content management, the ability of the computer to act as a library for digital content of all forms.

- Testing, the ability of the computer to pose challenges to an individual learner (or group of learners) and determine whether their response indicates understanding of the subject matter.

- Attention, the ability of the computer to assess the mental state of the user and to communicate this to a person, typically an instructor or tutor.

The last item is the weakest link. One of the greatest losses in adopting electronic methods for learning is the dramatic loss of feedback among the several parties on their mental and emotional states. In face-to-face interactions as humans we express these states through many visual signals. There are many attempts to compensate for this loss, for example through the use of bi-directional video or through animated avatars. This is a significant problem for e-Learning as skilled instructors make great use of the verbal and non-verbal feedback they receive from a face-to-face audience in adapting, repeating or restating information. The loss of that feedback mechanism is one of the great challenges that instructors using e-Learning face.

The first four capabilities are closely related to activities that have been demonstrated over many years to be key activities in enabling the learner to capture information and turn it into knowledge. What is new is that now the computer supports these activities. In recent years, e-learning practitioners, especially in corporate training, have recognised the need to apply all these various techniques in "blended learning".

Figure 1 illustrates blended learning. It chunks learning activities into four main layers - learning from information, learning from interaction, learning from collaboration, and learning from co-location (face-to-face). This provides a useful framework in which to place a variety of computersupported learning methods - it does not express a particular learning model. As we move vertically, we go from learner-led, individual-study, based on electronic media up to instructor-led, multiparty, face-to-face learning.

The left-hand side of Figure 1 focuses on "formal" learning; learning activities guided by an instructor towards some goal. Studies have recognised that learning does not stop with completion of the course, but continues through informal learning experiences. These ultimately deliver the majority of our working knowledge. We are concerned to provide computer support for these informal learning activities illustrated in the 
right-hand side of Figure 1. Moving horizontally, we go from formal, structured learning, often long-term or "Just-In-Case" learning usually performed in an office setting, to informal, unstructured, often short-term or "Just-In-Time" learning that may take place at any time or anywhere. Figure 1 summarises the range of formal and informal learning activities independent of pedagogy - for which we seek computer support. From this we can advance to asking what technologies are required to provide this support.

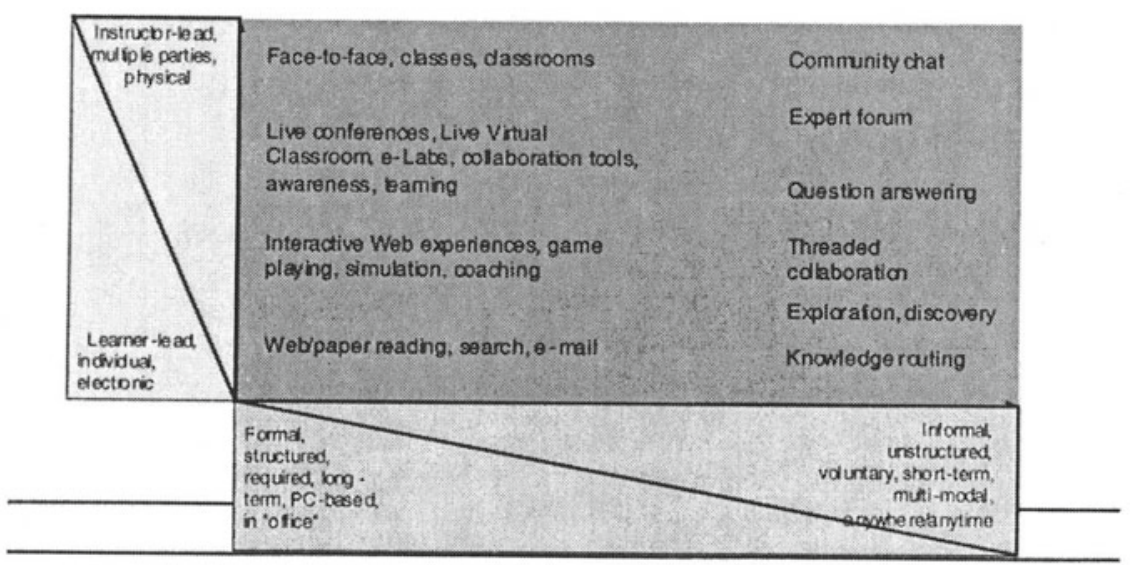

Figure 1. The blended learning model

Given this repertoire of capabilities can we develop prescriptive methods for combining these capabilities to compose learning activities that support a variety of teaching models? Such activities wrap around into the uppermost levels of the technology.

One last dimension is how the e-learning activity is delivered to the enduser. While desktop or laptop computers are the principal client devices, we anticipate that television will evolve into an interactive medium, capable of providing many, if not all capabilities discussed above. Networked game platforms (Sony PlayStation (C) and Microsoft X-Box ()) could, in principle, support these capabilities. Lastly, mobile devices such as PDAs, wearable computers, and $3 \mathrm{G}$ telephones may support sub-sets of these capabilities that have very high value for specific learning needs. 


\section{TECHNICAL FOUNDATIONS FOR E-LEARNING}

e-Learning systems exemplify distributed computing. Compared to many other forms of computing e-Learning has two special emphases - on content rather than transactions and on human-human interactions rather than purely human-machine interactions. Computer-based training pioneered many innovations in interactive systems, multi-media, and virtual reality. In many cases it has been too early in its adoption of these technologies and they have had to be implemented in the e-Learning applications, rather than being used to exploit facilities provided by the operating system or middleware. Consequently, earlier e-Learning systems have appeared to be a distinct class of computing, requiring its own unique technologies and standards.

However, e-Learning technology will soon converge with other contentcentric or interaction-centric activities to become "just" an instance of multiparty, network-mediated, adaptive, interactive, rich-media applications. Broadband distribution, digital media, XML composite "documents", collaboration, unstructured information management and digital rights management are all evolving to support these complex applications that eLearning needs. In response to broad-based needs, these technologies are on track to provide the primitives needed to implement e-Learning applications.

A key activity is ensuring that the requirements of e-Learning are incorporated in the relevant standards and implemented in products. We must partition these needs into the generic (requiring configuration to the activities in question) and the specific (to the activity). The e-Learning community must converge with these mainstreams and use commoditised technology. e-Learning will no longer be a distinct class of computing.

The driving force is the emergence of other multiparty, networkmediated, adaptive, interactive, rich-media applications - e-commerce systems that adapt their behaviour to the customer's preferences, negotiating or bidding systems that structure the dialogue among the parties, and games, a structured set of interactions among players. All of these specialise, but a key area of technology work lies in abstracting the generic mechanisms required to model these and other human-human and human-machine interactions. The emergence of these several forms of multiparty, networkmediated, adaptive, interactive, rich-media applications leads to the emergence of mainstream technologies that, in particular, are capable of supporting e-Learning. A clear, though unfortunate, example of this is the enormous overlap between metadata specifications for e-Learning Objects on the one hand and for interactive rich-media on the other (Learning Resources 2002; IEEE 2002; MPEG-7 2002). I refer to this as "convergence". 
Today we are close to a good set of distributed computing primitives based around open industry standards. We have ideas about how to assemble these into multi-party, network-mediated, adaptive, interactive, rich-media primitives for various purposes, including e-Learning (Figure 2). The foundations comprise the distributed computing primitives that are generally accepted as common elements for almost all the mainstream applications. However we should be trying to push convergence as high up the stack as possible. We do not need a multiparty collaboration primitive specialised on e-Learning but we need a generic multiparty collaboration primitive that could be configured to support various learning interactions.

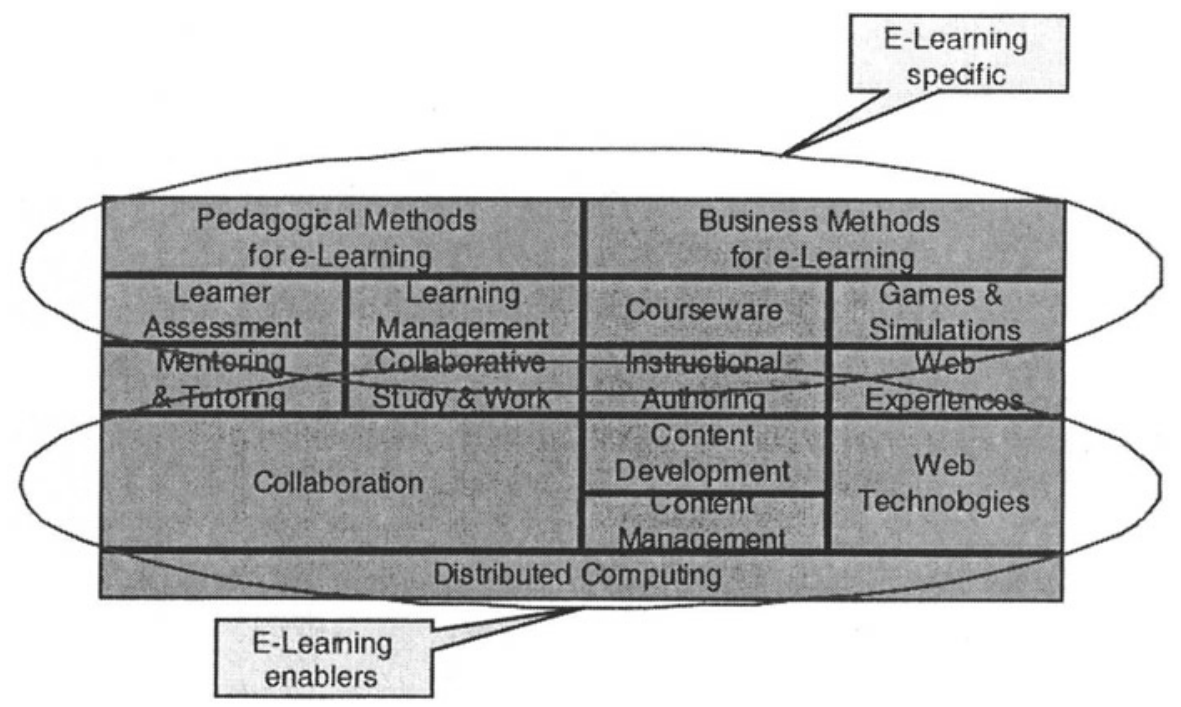

Figure 2. Building blocks for e-Learning

Above the Distributed Computing layer lies convergent middleware that provides support for generic multiparty, network-mediated, adaptive, interactive, rich-media applications. This convergent middleware includes collaboration, content development, content management, and Web technologies. Above this layer are e-Learning-specific applications that leverage other primitives to compose complex applications that support pedagogically sound learning activities.

We require research programs that bring together Computer Science (CS) and Human Computer Interface (HCI) people working bottom up to study how to generalise the application of the primitives into bigger chunks that 
can be configured to represent, say, e-Learning, e-Commerce, e-Negotiation or e-Games.

If we take a top down view, there is work in which we ask practitioners instructors, tutors and mentors - to engage with the CS/HCI community on how to most effectively apply these chunks to model electronically their best behavioural practices. That is, models of interaction, spanning the two dimensions of blended learning shown in Figure 1, and supporting a variety of pedagogical principles. These models act as maps not rigid processes illustrating ways the learner may reach the learning goal. The learner, guided additionally by an instructor, a coach, or peers may explore and follow any route. Each domain: learning, commerce or negotiation has half a century of behavioural research behind it that is collected into a finite set of models/principles - discovery-based learning, inquiry-based learning and cognitive apprenticeship.

I do not believe that it is the job of the e-Learning technology community to figure which model is best: that role belongs to the instructors. Our job is to figure out how the chunked primitives should be configured to support each of these models.

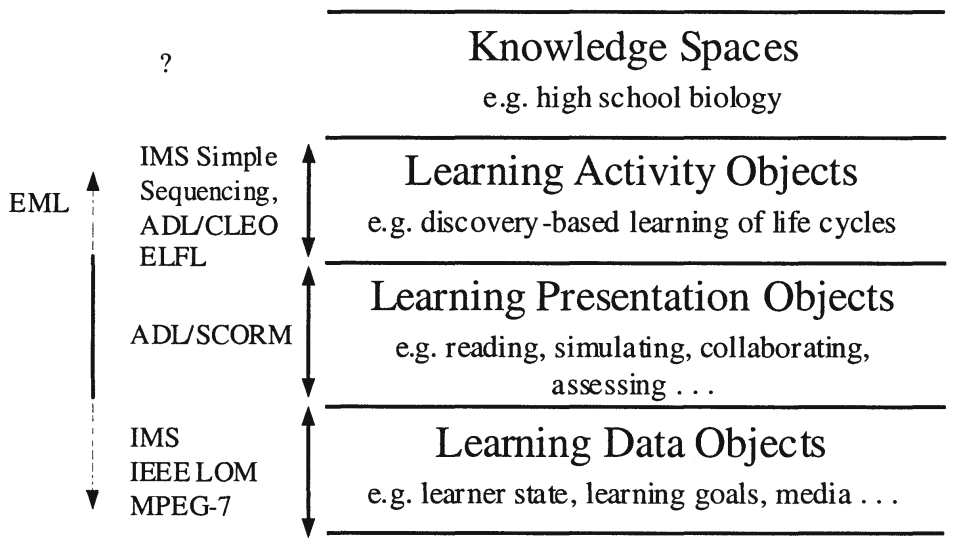

Figure 3. e-Learning structures showing the hierarchy of learning objects

We must judge what is most effective, since inevitably when we transfer these models to electronic interaction they change subtly. We must work 
with the practitioners to determine how to improve fidelity to the original models or exploit the new characteristics. Let us first develop the capability building on the work of the Educational Modelling Language (EML) group at the Open University of the Netherlands.

In addition to functional support for the delivery of learning activities, we also need technology support for structuring of digital content that creates these experiences. Figure 3 shows a hierarchy of such structures, commonly referred to as learning objects. At the base are the data primitives of eLearning: rich-media content, learner state and learning objectives (Learning Data Objects in our terminology). In the second layer these are composed into short learning experiences (units of study in EML terminology Learning Presentation Objects in ours). The third layer is the composition of learning experiences into extended learning experiences, lasting for an entire session, class, or course. In the topmost level there is the structuring of knowledge itself. Figure 3 also shows the coverage of various learning object standards over these layers.

Structuring Learning Data Objects is the basic digital library problem: annotating digital content with metadata that allows it to be stored and retrieved. The IEEE Learning Object Metadata (LOM) standard has recently been adopted for this purpose (IEEE 2002). Structuring Learning Data Objects into Learning Presentation Objects is addressed by both the EML (Koper 2001) and Shareable Content Object Reference Model (SCORM) (ADL 2002) specifications from the Advanced Distributed Learning Network (ADL). The IEEE LOM standard and the ADL SCORM specification are widely adopted in commercial learning systems.

The structuring of learning experiences (Learning Presentation Objects or EML Units of Study) into extended learning activities (Learning Activity Objects) is an active area of exploration. This is being approached in terms of sequencing Learning Presentation Objects into graphs whose nodes are the learning experiences. The exit from each node - at the completion of the learning experience - is connected to one or more subsequent nodes. The selection of which subsequent node is visited may be made to depend on inline assessment or other learner-centric factors.

One element missing from these specifications and standards is a strong architectural model. In our work we consider both the structuring of multiparty, network-mediated, adaptive, interactive, rich-media learning activities and the end-to-end delivery platform as well as the content lifecycle. Our project on the sequencing problem is known as the e-Learning Flow Language (ELFL) (Farrell 2002). It adds to the existing Learning Data Objects and Learning Presentation Objects the concept of Learning Control Objects. Their function is to enable the control of the flow of learning experience across the graph of Learning Presentation Objects. These 
varying paths may be made dependent on such factors as the learner's prior knowledge, the learner's role (practitioner, manager or inspector), or the learning need ("Just-In-Case" or "Just-In-Time"). Each node or learning experience is modelled as a Web Service, which connects us to a rich set of distributed computing primitives, such as the Web Services Description Language (Web Services 2002).

We have reviewed this direction of research with IMS, ADL and academic researchers. It is our intent - when we have working code - to introduce these concepts into the open industry standards processes.

Above these models of interaction there is the background or context for the learning activity. A key goal of deep learning is not merely the memorisation of information, but the generation of the mental framework or pigeonholes of a particular discipline into which new information can be rapidly assimilated. This is often referred to as a "mental map" - from the point of view of a person - or "knowledge map" from the more abstract view of the discipline itself. One traditional tool for representing the knowledge map is a book, which presents the information on say, physics, in a logical sequence that can be comprehended by the learner. Another common knowledge map representation is the organisation of books in a library.

A syllabus can be represented as a taxonomy or a graph. This may be a good mechanism for structuring a collection of learning activities that provide syllabus coverage. A graph could include constraints in the form of prerequisites to launching a given activity but a graphical representation of this kind may not be the most engaging or memorable way of conveying the structure of the knowledge space.

What kind of computer-supported modelling can we apply to help the learner develop the mental map? Such work invariably involves various ways of visualising the relationships among information elements of a discipline like ontological representations of concepts, virtual reality spaces, and adaptations of real spaces (Sherman 2002; Cetkovic et al 2002).

Lastly we need a set of authoring tools that enable practitioners to manipulate these electronic models in terms of the models of their own profession. These tools must provide support for the practitioner at four levels:

- finding the learning data - the basic information;

- composing this into a meaningful and engaging learning activity according to some pedagogical principles;

- sequencing learning experiences into an overall set of learning activities to cover a syllabus;

- integrating several learning activities into a visualisation of the knowledge space. 
This is a very difficult problem. Today we can do a poor-to-fair job on finding the learning data and we have template-based tools for composing these into learning experiences. Better support for pedagogy in creating learning experiences is a frequent customer request. We have little or no idea of how to use the emerging sequencing techniques. Despite experimentation on visualising knowledge maps there is no codification of how to effectively apply these. If this seems hard from the perspective of technology support, it appears incomprehensible from the perspective of most practitioners. No wonder they are confused about learning objects. Practitioners and technologists together need to do a lot of exploration and codification of how to use these new structuring methods. Then we have to make these available in terms of concepts that are meaningful to the practitioners through tools that are as easy to learn to use badly as Microsoft Powerpoint ().

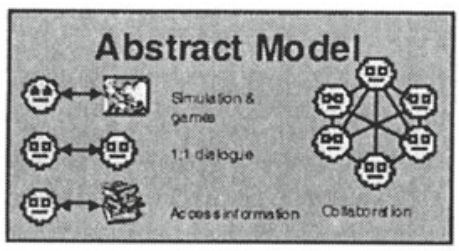
Standards-based methods for representing single party or multi-party interactions as data, not code
$\square$ Run-time environment for interactions, separated from the content
$\square$ Start with learning
$\square$ Generalize into tools for web-based experiences

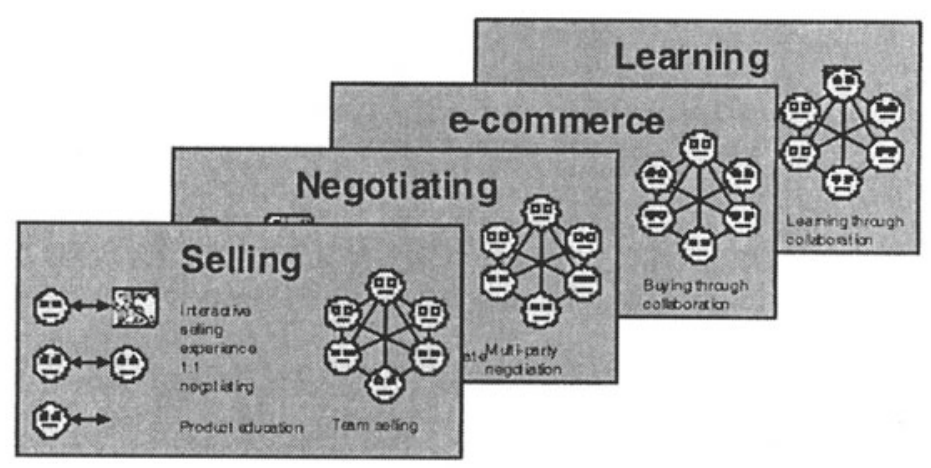

Figure 4. Modelling human activities

Fortunately we are not alone. The model we have for the application of computers in support of learning is that of a collection of mechanisms for enabling interaction among one or more learners, an instructor, a body of information, a group of peers, and possibly a coach. This is a very finite set of activities. I earlier indicated a number of fronts where Computer Science is intersecting with Behavioural Science with the aim of modelling human behaviour. In e-commerce we seek to make Web sites that are less 
frustrating to use because their behaviour conforms to the user's mental task model. In negotiation we model the progressive disclosure of information between, say, a safe driver needing insurance and one or more insurance providers. As Figure 4 shows, in the next years we expect to develop generalised methods for supporting these kinds of behavioural modelling which can then be specialised to an area such as e-Learning. This is important. It finally connects e-Learning techniques and technologies to the mainstream of commercial software development. This will attract the benefit of a much larger investment stream.

We believe the key areas for technologies for delivering the e-Learning experience in the short term are:

- Ensuring that mainstream, converged tools and middleware support the generic requirements of e-Learning as converged primitives in areas such as content management, collaboration and authoring.

- Separating out the purely e-Learning concerns and developing ways to express these through lower-level, converged primitives. We see ELFL as an example of this - leveraging Web Services primitives to create educational experiences.

- Bridging the semantic gap between low-level content manipulation and high-level lesson/course design. The authoring burden on e-Learning practitioners is extremely high. To fulfil even the more modest visions, we must find ways to enable instructors to focus on the problems of learning and to use e-Learning tools in that context.

\section{SUMMARY}

Our view of the role of computers in learning is that they provide a repertoire of capabilities from interacting with information through simulated experiences to collaborative problem solving and coaching. These capabilities are rooted in the mainstream technologies of distributed computing which are being used to support relatively new forms of computing applications that are intensive in the use of rich-media content and in structured interactions both human-human and human-machine. These roots endow these capabilities with potentially revolutionary attributes. These attributes include transcending time and space and enabling new forms of scaling. They may enable teaching models to scale to one to thousands at one extreme and 1 to 1 at the other extreme. The technical potential is very real. The infrastructure to deliver is being put in place. How to make it effectively useful to practitioners emerges as the next challenge. 
This new class of multiparty, network-mediated, adaptive, interactive, rich-media applications aims at areas such as e-Learning, e-commerce, gaming, and other areas where the quality of the end-user experience is a critical metric of the effectiveness of the application. The spread of these requirements into several areas will drive common functionality - such as adaptation or structured interaction modelling - into mainstream middleware. e-Learning technology then becomes the configuration and structuring of this middleware and related content to select from the repertoire of capabilities those that will provide the needed experience. The focus of recent work has been the generation of specifications for structuring richmedia content for this purpose. We are moving onto research in representing structured, adaptive interactions and various forms of collaboration.

The most challenging work lies in finding means to enable practitioners to make the composition and use of computer-based experiences part of their everyday work. This will require adaptation on their part and a major effort by technologists to bridge the conceptual gap between the technology model and the practitioners' models. Success here has the potential to lead to major transformation, if not revolution, in the delivery of education and training.

The technologists are already working with specialists in pedagogy, with educational policy makers, with behaviourists and other members of the soft sciences. I believe we need to engage with the practitioners, both to understand better how these techniques fit into the practices of training and education today and also to finally bring clarity and focus to my opening question: What critical problems in training and education can we solve through the use of computers?

\section{REFERENCES}

Advanced Distributed Learning Network (2002) ADL SCORM specification. [http://www.adlnet.org]

Cetkovic, A., Drexler, H., Guinand, M., Jauslin, D. and Leummens, M. (2002) [http://www.ethworld.ethz.ch/nw/competition/details/?tarnum=3007]

Farrell, R. (2002) Dialogic Engagement for Active Web-based Learning. In E-Learn 2002 World Conference on E-Learning in Corporate, Government, Healthcare and Higher Education. Montreal, Canada. In press.

IEEE P1484.12 Learning Object Metadata Working Group.

[http://ltsc.ieee.org/wg12/]

Koper, R. (2001) Educational Modelling Language. Open University of the Netherlands [http://eml.ou.nl/introduction/docs/ped-metamodel.pdf]

Learning Resource Meta-data Specification (2002) IMS Global Learning Consortium. [http://www.imsproject.org/metadata/]

MPEG-7. [http://mpeg.telecomitalialab.com/standards/mpeg-7/mpeg-7.htm] 
Sherman, R. A. (2002) Abstraction in Concept Map and Coupled Outline Knowledge

Representations. In Journal of Interactive Learning Research. In press.

Web Services Description Language (2002) [http://www.w3.org/TR/wsdl] 